\title{
Improvement in coronary anastomosis with cardiac surgery simulation
}

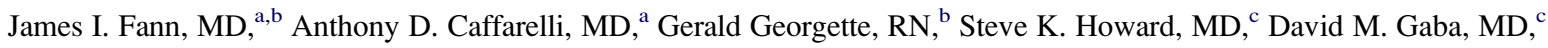 \\ Patricia Youngblood, PhD, ${ }^{\mathrm{d}} \mathrm{R}$. Scott Mitchell, MD, ${ }^{\mathrm{b}}$ and Thomas A. Burdon, MD ${ }^{\mathrm{a}, \mathrm{b}}$
}

\begin{abstract}
Objective: Cardiac surgery trainees might benefit from simulation training in coronary anastomosis and more advanced procedures. We evaluated distributed practice using a portable task station and experience on a beating-heart model in training coronary anastomosis.
\end{abstract}

\begin{abstract}
Methods: Eight cardiothoracic surgery residents performed 2 end-to-side anastomoses with the task station, followed by 2 end-to-side anastomoses to the left anterior descending artery by using the beating-heart model at 70 beats/min. Residents took home the task station, recording practice times. At 1 week, residents performed 2 anastomoses on the task station and 2 anastomoses on the beating-heart model. Performances of the anastomosis were timed and reviewed.
\end{abstract}

Results: Times to completion for anastomosis on the task station decreased $20 \%$ after 1 week of practice (351 \pm 111 to $281 \pm 53$ seconds, $P=.07$ ), with 2 residents showing no improvement. Times to completion for beating-heart anastomosis decreased $15 \%$ at 1 week (426 \pm 115 to $362 \pm 94$ seconds, $P=.03$ ), with 2 residents demonstrating no improvement. Home practice time (90-540 minutes) did not correlate with the degree of improvement. Performance rating scores showed an improvement in all components. Eighty-eight percent of residents agreed that the task station is a good method of training, and $100 \%$ agreed that the beating-heart model is a good method of training.

Conclusions: In general, distributed practice with the task station resulted in improvement in the ability to perform an anastomosis, as assessed by times to completion and performance ratings, not only with the task station but also with the beating-heart model. Not all residents improved, which is consistent with a "ceiling effect"' with the simulator and a "plateau effect" with the trainee. Simulation can be useful in preparing residents for coronary anastomosis and can provide an opportunity to identify the need and methods for remediation.

Changes in surgical training, partly in response to patient safety concerns, financial pressures, and resident work hour limitations, have compelled surgical educators to evaluate more effective methods of teaching psychomotor skills. ${ }^{1-20}$ Assisting with coronary artery bypass grafting supervised by attending staff is the current method of training for coronary anastomosis; however, the trainee might benefit from simulation training in this and more advanced procedures. ${ }^{17-20}$ Technical challenges of beating-heart surgery include coronary artery stabilization and performing accurate and expeditious anastomoses on moving target vessels. Non-beating-heart and beating-heart simulators and porcine heart models can provide visual and tactile feedback in performing coronary anastomoses and instill confidence in the participants. ${ }^{17-20}$ Simulators need to provide a realistic and

From the Departments of Cardiothoracic Surgery ${ }^{\mathrm{a}}$ and Anesthesiology ${ }^{\mathrm{c}}$ and SUMMIT, ${ }^{\mathrm{d}}$ Stanford University, Stanford, Calif, and the Cardiothoracic Surgery Section, ${ }^{\mathrm{b}}$ VA Palo Alto Health Care System, Palo Alto, Calif.

This study was supported in part by the WTSA Doty Award from Medtronic, Inc, and a grant from Carbomedics of the Sorin Group.

Received for publication May 15, 2008; revisions received July 17, 2008; accepted for publication Aug 7, 2008.

Address for reprints: James I. Fann, MD, Department of Cardiothoracic Surgery, Stanford University, 300 Pasteur Dr, Stanford, CA 94305 (E-mail: jfann@stanford.edu). J Thorac Cardiovasc Surg 2008; 136:1486-91

$0022-5223 / \$ 34.00$

Copyright (c) 2008 by The American Association for Thoracic Surgery doi:10.1016/j.jtcvs.2008.08.016 graduated training experience and have valid educational objectives; they also should be cost-effective and of relatively low maintenance. Of value would be a component of simulation that allows the trainee to practice on his or her own time instead of being constrained by work hour limitations and availability of animal laboratory facilities.

To date, there are limited data regarding the effectiveness of simulators for coronary anastomosis. We have developed a series of task stations and procedures that are intended to provide initial and follow-up training and practice of routine and complex cardiac surgical procedures for the resident. In this study we evaluated distributed practice (ie, practice interspersed with periods of rest) using a portable task station and the experience with the beating-heart model in training techniques of coronary anastomosis.

\section{MATERIALS AND METHODS}

Eight cardiothoracic surgery residents, all trained in general surgery, participated. The residents' experience ranged from no previous formal cardiac surgery experience to those with at least 2 years of cardiac surgery training; 3 trainees were in the first year of training, 2 were in the second year, and 3 were in the third year. Approval was obtained from the institutional review board.

\section{Cardiac Surgical Simulation Laboratory}

The laboratory is configured to be similar to an operating room (Figure 1). It is equipped with an operating table with an overhead light, 


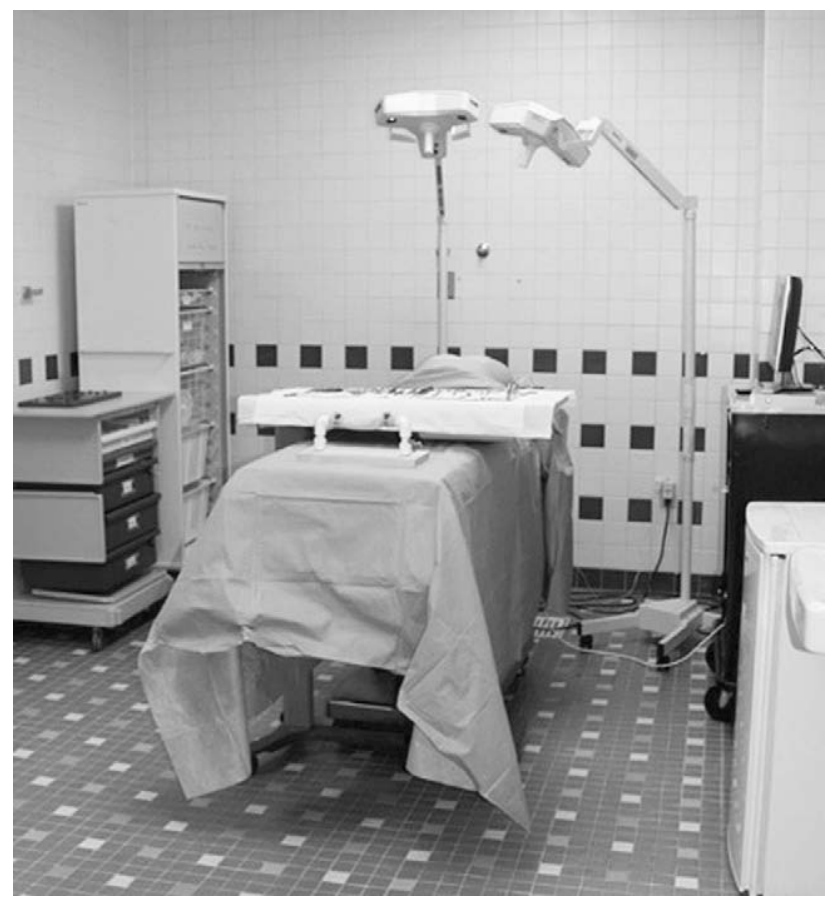

FIGURE 1. The cardiac surgery simulation laboratory is configured to be similar to an operating room.

instrument/supply cart, video cameras and monitors, wet-laboratory stations, and task stations, including anastomosis stations with synthetic vessels, heart models with disposable coronary arteries, and a beating-heart station.

\section{Anastomosis Task Station and Beating-heart Model}

The anastomosis task station provides the resident with a portable apparatus to practice end-to-side anastomosis (Figure 2). Mounted on the portable task station are silicone target vessels, which are $3 \mathrm{~mm}$ in diameter, as are the silicone vein grafts (Chamberlain Group, Great Barrington, Mass). The surgical instruments are identical to those used in the clinical operating room, and the suture used is 6-0 polypropylene.

The beating-heart model is constructed of silicone and connected to a controller and external compressor (Chamberlain Group). The compressor is connected to the tubing inside the heart through the controller via 2 solenoid valves, one that controls the squeezing of the heart and one that controls the apex-to-atria pull-up. They are offset fractionally in time to add to the rocking motion. Partially embedded in the myocardium are 2-mm target coronary arteries (left anterior descending artery, obtuse marginal artery, and posterior descending artery). The heart is placed in a plastic torso simulating the pericardial well (Figure 3). The controller permits changes in heart rate from 50 to 90 beats/min.

\section{Study Protocol}

This study evaluated the utility of the portable anastomosis task station and beating-heart models and the effectiveness of distributed practice. In the simulated operating room the resident was instructed on the setup of the task station and the beating-heart model by an attending surgeon. A 5minute instructional video recording demonstrating the use of the Castroviejo needle driver was viewed by the resident. Within a 30-minute period, the resident performed 2 end-to-side anastomoses of synthetic vein graft to target vessel using the task station. The use of the task station was followed by 2 end-to-side anastomoses to the left anterior descending artery by using the beating-heart model at 70 beats/min. The resident took home the task
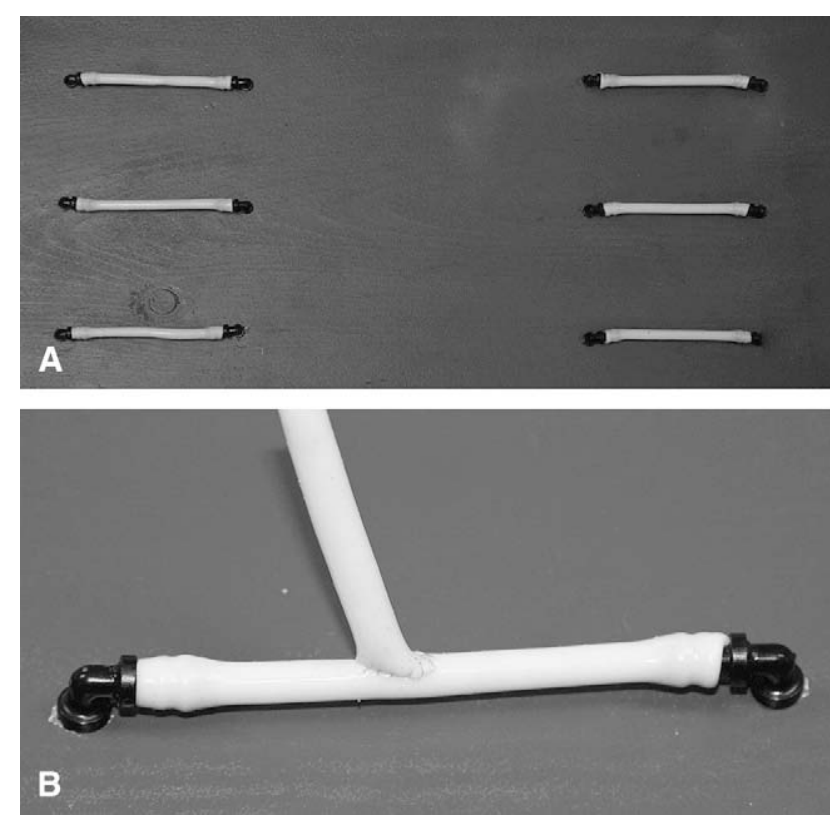

FIGURE 2. The portable anastomosis task station has mounted six 3-mm synthetic target vessels (A), which permit multiple end-to-side anastomoses (B).

station to practice, recording practice time in minutes. At 1 week, the resident returned to the simulated operating room and performed 2 anastomoses on the task station and 2 anastomoses on the beating-heart model, identical to the initial session. Performance of the anastomosis with the task station and the beating-heart model was timed in seconds, recorded with a digital video camera, and reviewed by 2 experienced surgeons using performance and overall rating scores.

\section{Performance Assessment}

All anastomoses from the task station and beating-heart model were excised and evaluated for patency. The video data were converted to mpegs, stored on the computer hard disk, and reviewed by 2 experienced surgeons in a blinded fashion; the reviewing surgeons were instructed and anchored in the use of the rating scores. The resident's performance was

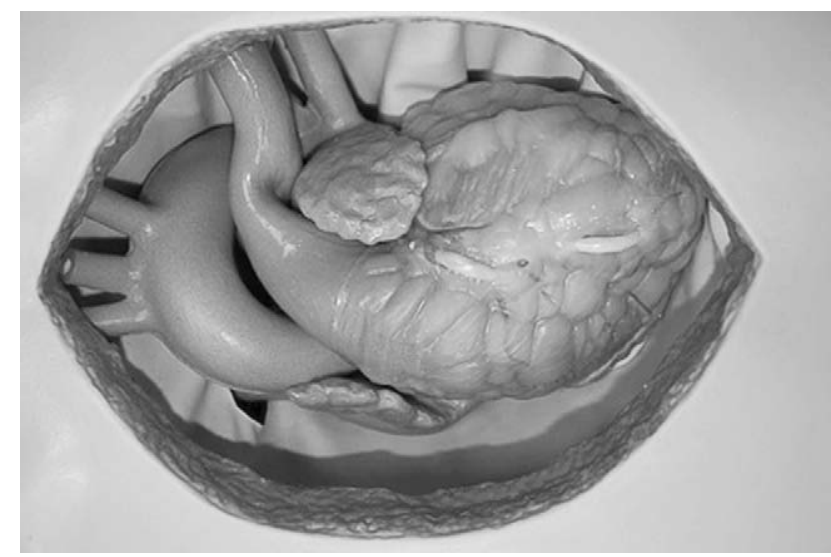

FIGURE 3. The beating-heart model is constructed of silicone and connected to a controller and external compressor. Partially embedded in the myocardium are 2-mm target coronary arteries. The heart is placed in a plastic torso simulating the pericardial well. 
TABLE 1. Components of performance rating scores

1. Graft orientation (proper orientation for toe-heel, appropriate start and end points)

2. Bite appropriate (entry and exit points, number of $\begin{array}{lllll}1 & 2 & 3 & 4 & 5\end{array}$ punctures, even and consistent distance from edge)

3. Spacing appropriate (even spacing, consistent distance from previous bite, too close vs too far)

4. Use of Castroviejo needle holder (finger placement, $\begin{array}{llllll}1 & 2 & 3 & 4 & 5\end{array}$ instrument rotation, facility, needle placement, pronation and supination, proper finger and hand motion, lack of wrist motion)

5. Use of forceps (facility, hand motion, assist needle $\quad \begin{array}{lllll}1 & 2 & 3 & 4 & 5\end{array}$ placement, appropriate traction on tissue)

6. Needle angles (proper angle relative to tissue and $\begin{array}{llllll}1 & 2 & 3 & 4 & 5\end{array}$ needle holder, consider depth of field, anticipating subsequent angles)

7. Needle transfer (needle placement and preparation from stitch to stitch, use of instrument and hand to mount needle)

8. Suture management/tension (too loose vs too tight, use tension to assist exposure, avoid entanglement)

9. Knot tying (adequate tension, facility, finger and hand follow for deep knots)

Scores: 1, excellent, able to accomplish goal without hesitation, showing excellent progress and flow; 2 , good, able to accomplish goal deliberately, with minimal hesitation, showing good progress and flow; 3 , average, able to accomplish goal with hesitation, discontinuous progress and flow; 4 , below average, able to partially accomplish goal with hesitation; 5 , poor, unable to accomplish goal, marked hesitation (adapted from the Objective Structured Assessment of Technical Skill ${ }^{3}$ ).

assessed by using performance rating scores, consisting of different aspects of surgical skills rated on a 5-point scale (Table 1) and modified from the Objective Structured Assessment of Technical Skills described by Reznick and colleagues. ${ }^{3}$ The overall rating score is a 3-point scale (1, good; 2, average; 3 , poor) based on the general set-up and surgeon positioning, graft and target orientation, instrument handling, tissue/material handling, needle handling, general motion, flow of task, and forward planning.

\section{Residents' Rating (Exit Questionnaire)}

After completion of the protocol, the participants were asked to complete an exit questionnaire consisting of 8 statements scores as follows: " agree," "'somewhat agree," "'not sure," "'somewhat disagree," and "disagree." The purpose of the questionnaire was to assess the residents' opinions on the realism of the simulation tasks, the efficacy of the simulator training experience, and their confidence in performing the surgical procedures after simulator training:

1. The vessels on the anastomosis task station are realistic.

2. The beating-heart model is as realistic as an actual beating heart.

3. Performing an anastomosis on the task station is as real as an actual anastomosis.

4. Performing an anastomosis on the beating-heart model is a realistic representation of the procedure.

5. The anastomosis task station is a good method of training technical skills.

6. The beating-heart model is a good method of training technical skills.

7. I am more confident in coronary anastomosis.

8. I am more confident in beating-heart coronary anastomosis.

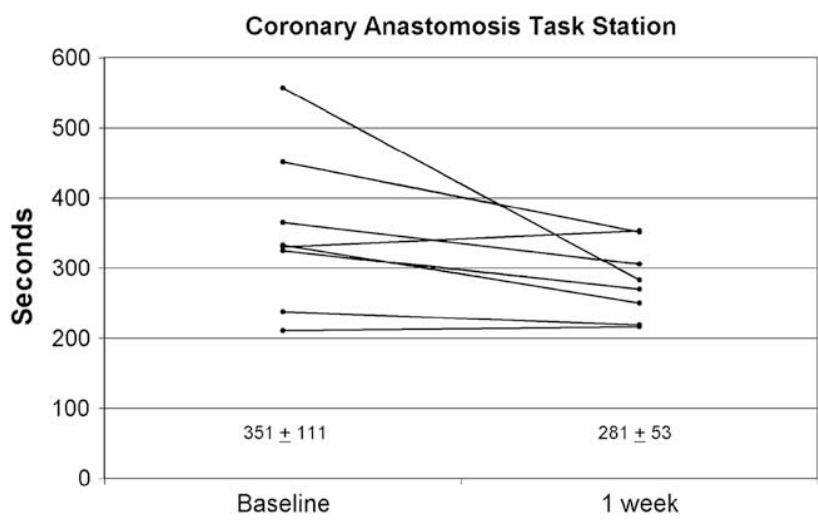

FIGURE 4. Times to completion before and after practice for each resident using the anastomosis task station.

\section{Data Analysis}

The data were analyzed by using paired $t$ tests to compare baseline times to completion and those after 1 week of practice for the task station and those at 1 week for the beating-heart model. To assess the interrater reliability when scoring the participants, we used the statistic Savr described by Gaba and associates. ${ }^{21}$ Savr is a variant of Sav, which is the most generalized form of the $\kappa$-like statistics of interrater agreement referenced to chance. Savr takes into account the ordinal nature of the scale and can accommodate 2 or more raters. For Savr, the by-chance reference is computed on the assumption that raters would have an equal chance of using any of the rating scale elements in rating any particular item and subject. Savr was calculated for each performance rating score and overall rating score. Correlation coefficients for home practice times and times to completion of the anastomosis task station were obtained (PRISM 4 for MacIntosh; GraphPad Software, Inc, La Jolla, Calif).

\section{RESULTS}

\section{Anastomosis Times}

The times to completion for each anastomosis on the task station decreased $20 \%$ overall after 1 week of practice, from $351 \pm 111$ seconds (range, $211-557$ seconds) to $281 \pm 53$ seconds (range, 216-353 seconds; $P=.07$; Figure 4). Two residents had no improvement using the anastomosis task station; one was in the third year of training and one was in the first year of training. For the third-year resident, the times to completion were 211 seconds before practice to 216 seconds after practice, which were the lowest times in this study. One resident at the first-year level had a slight increase in time (331 before practice to 353 seconds after practice) using the anastomosis task station. For all thirdyear residents, the range of times to completion were between 216 and 270 seconds after practice, which were in general lower than those of the first- and second-year residents.

The times to completion for each beating-heart anastomosis decreased $15 \%$ at 1 week, from $426 \pm 115$ seconds (range, 269-570 seconds) to $362 \pm 94$ seconds (range, 221-535 seconds; $P=.03$; Figure 5). Two residents (1 at the first-year level and 1 at the second-year level) demonstrated no improvement using the beating-heart model; the 


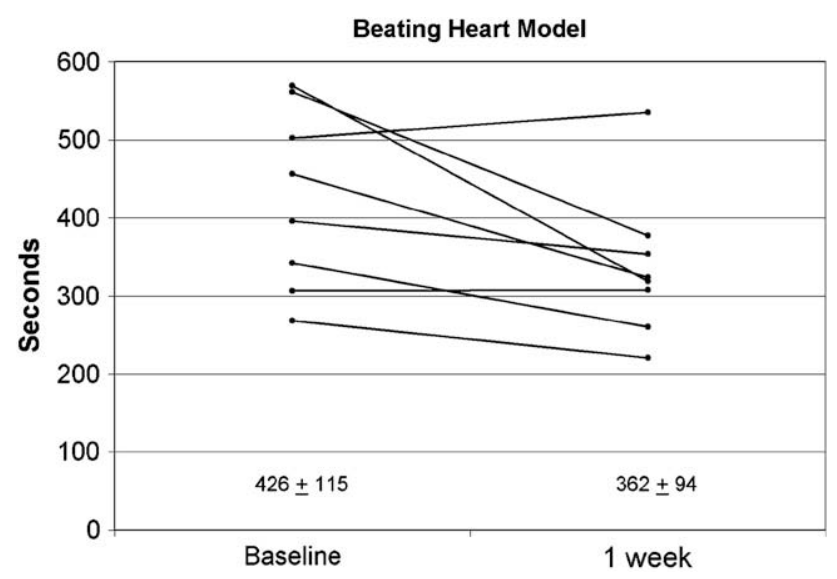

FIGURE 5. Times to completion before and after practice for each resident using the beating-heart model.

first-year resident required a relatively longer period for both prepractice and postpractice sessions (503 seconds before practice to 535 seconds after practice). The second-year resident had moderately low times for both prepractice and postpractice sessions (307 seconds before practice to 308 seconds after practice).

Home practice using the task station ranged from 90 to 540 minutes, with the first-year residents practicing between 150 and 540 minutes (distributed over 5-7 days), the second-year residents practicing between 100 and 225 minutes (3-5 days), and the third-year residents practicing between 35 and 90 minutes (3-4 days). There was no correlation between total practice time and improvement in performance, as measured by time to completion $(r=.48, P=.23)$ or days of practice and time to completion $(r=0.386, P=.35)$.

\section{Technical Skills Assessment}

The anastomoses from the task station and the beatingheart model were assessed and noted to be patent in all cases, with no decrease in quality of the anastomoses. Performance rating scores showed an overall improvement in all components (Table 2). For the anastomosis task station, the most improvement was seen in suture management and tension. For the beating-heart model, the most improvement was again seen in suture management and tension.

For the overall rating score, the mean for the task station before practice was $1.6 \pm 0.6$; for the postpractice session, it was $1.3 \pm 0.5$. For the beating-heart model, the overall rating score before practice was $1.6 \pm 0.5$; for the postpractice session, it was $1.2 \pm 0.6$. For the task station and beating-heart model, 2 residents ( 1 first-year resident and 1 second-year resident) achieved good scores (ie, $1=$ good) at prepractice and postpractice sessions and therefore demonstrated no improvement.

The interrater reliability between the 2 surgeon reviewers for the performance rating scores and overall rating scores
TABLE 2. Mean performance rating scores comparing scores before and after practice

\begin{tabular}{|c|c|c|c|c|}
\hline & \multicolumn{2}{|c|}{ Anastomosis task station } & \multicolumn{2}{|c|}{ Beating-heart model } \\
\hline & Before & After & Before & After \\
\hline 1. Graft orientation & $2.1 \pm 1.5$ & $1.4 \pm 0.8$ & $1.8 \pm 1.1$ & $1.4 \pm 0.7$ \\
\hline 2. Bite appropriate & $2.0 \pm 1.0$ & $1.5 \pm 0.8$ & $1.7 \pm 0.8$ & $1.3 \pm 0.7$ \\
\hline 3. Spacing appropriate & $1.9 \pm 0.9$ & $1.4 \pm 0.7$ & $1.7 \pm 0.8$ & $1.3 \pm 0.7$ \\
\hline $\begin{array}{l}\text { 4. Castroviejo needle } \\
\text { holder use }\end{array}$ & $2.0 \pm 1.4$ & $1.7 \pm 1.0$ & $1.8 \pm 1.0$ & $1.6 \pm 1.3$ \\
\hline 5. Use of forceps & $2.2 \pm 1.1$ & $2.0 \pm 1.3$ & $2.1 \pm 1.0$ & $1.6 \pm 0.9$ \\
\hline 6. Needle angles & $1.8 \pm 1.0$ & $1.4 \pm 0.7$ & $1.8 \pm 0.9$ & $1.5 \pm 1.1$ \\
\hline 7. Needle transfer & $2.2 \pm 1.1$ & $1.6 \pm 0.9$ & $2.1 \pm 1.2$ & $1.6 \pm 1.2$ \\
\hline $\begin{array}{l}\text { 8. Suture } \\
\text { management/tension }\end{array}$ & $2.2 \pm 1.2$ & $1.4 \pm 0.7$ & $1.8 \pm 0.9$ & $1.3 \pm 0.7$ \\
\hline 9. Knot tying & $1.6 \pm 0.9$ & $1.4 \pm 0.7$ & $1.8 \pm 0.9$ & $1.4 \pm 0.7$ \\
\hline
\end{tabular}

Data are expressed as means \pm standard deviation.

for the task station and for the beating-heart model was 0.88 , demonstrating good reliability.

\section{Residents' Ratings}

The exit questionnaire showed $88 \%$ of the residents agreed (and 12\% somewhat agreed) that the anastomosis task station is a good method of training technical skills; $100 \%$ agreed that the beating-heart model is a good method of training (Table 3). Sixty-three percent agreed or somewhat agreed that performing an anastomosis with the task station was realistic; $75 \%$ agreed or somewhat agreed that an anastomosis on the beating-heart model was realistic. Regarding the degree of realism of the synthetic model and graft material, $63 \%$ of the residents somewhat agreed that the anastomosis task station was realistic; for the beatingheart model, $88 \%$ agreed or somewhat agreed that the model was realistic. Finally, $100 \%$ of the residents either agreed or somewhat agreed they felt more confident in performing a coronary anastomosis after using the task station; on the other hand, only $50 \%$ of the residents felt more confident (agreed or somewhat agreed) with beating-heart surgery.

\section{DISCUSSION}

In general, distributed practice with the task station resulted in improvement in the ability to perform an anastomosis, as assessed by time to completion and performance rating scores, not only with the task station but also with the beating-heart model. Not all residents improved, particularly with the task station, which is consistent with a "ceiling effect" with the simulator, a "plateau effect" with the trainee, or both. ${ }^{6,11,16}$ Simulation might be useful in preparing residents for coronary anastomosis in the clinical setting and might provide an opportunity to identify the need and methods for remediation.

Cognitive and technical learning in the operating room provides little opportunity for practice and reflection; therefore simulation can provide needed training and practice 
TABLE 3. Resident exit questionnaire

\begin{tabular}{|c|c|c|c|c|c|}
\hline Statement & Agree & Somewhat agree & Not sure & Somewhat disagree & Disagree \\
\hline 1. The vessels on the anastomosis task station are realistic. & - & $5(63 \%)$ & $1(13 \%)$ & $2(25 \%)$ & - \\
\hline 2. The beating-heart model is as realistic as an actual beating heart. & $2(25 \%)$ & $5(63 \%)$ & $1(13 \%)$ & - & - \\
\hline $\begin{array}{l}\text { 3. Performing an anastomosis on the task station is as real as an actual } \\
\text { anastomosis. }\end{array}$ & $2(25 \%)$ & $3(38 \%)$ & $1(13 \%)$ & $2(25 \%)$ & - \\
\hline $\begin{array}{l}\text { 4. Performing an anastomosis on the beating-heart model is a realistic } \\
\text { representation of the procedure. }\end{array}$ & $3(38 \%)$ & $3(38 \%)$ & $2(25 \%)$ & - & - \\
\hline $\begin{array}{l}\text { 5. The anastomosis task station is a good method of training technical } \\
\text { skills. }\end{array}$ & $7(88 \%)$ & $1(13 \%)$ & - & - & - \\
\hline 6. The beating-heart model is a good method of training technical skills. & $8(100 \%)$ & - & - & - & - \\
\hline 7. I am more confident in coronary anastomosis. & $6(75 \%)$ & $2(25 \%)$ & - & - & - \\
\hline 8. I am more confident in beating-heart coronary anastomosis. & $2(25 \%)$ & $2(25 \%)$ & $3(50 \%)$ & - & - \\
\hline
\end{tabular}

outside the operating room. ${ }^{1-16}$ Although effective in the past, training in coronary anastomosis based on supervised operating with attending staff might not be appropriate in the current educational environment. The use of live-animal models to gain proficiency in surgical skills is costly and not generally accepted because of legal and ethical concerns. $^{2,3,7,19}$ Other investigators have used beating-heart simulators with synthetic and tissue grafts and noted that the participants were more confident in their ability to perform anastomoses on the beating heart. ${ }^{17-19}$ Advantages of existing simulation models include relatively realistic appearance, ability to expose technical deficiencies, and learning exposure maneuvers. Ramphal and coworkers ${ }^{20}$ developed an explanted porcine heart model in a simulated operating room environment with hemodynamic monitoring. This realistic simulator was used for training in coronary artery bypass grafting and valvular procedures. Acknowledged drawbacks of this model might be that the preparation is time consuming and the simulation exercise might take as long as an actual operation. Also, both resident and surgical staff must have dedicated time for such simulation, which is not portable and cannot be taken home as "homework."

Much work has been done in the development of performance metrics in using surgical training models and whether skills and tasks taught in laboratory environments can translate to the clinical setting. ${ }^{8,10,11,13-15}$ Based on the learning principle of massed versus distributed practice in the domains of psychology, athletics, and surgical laparoscopy, distributed practice appears to lead to better skill acquisition and retention. ${ }^{8,9,22}$ Thus our priority in establishing a cardiac surgery simulation curriculum has been to provide the resident with laboratory models that can replicate surgical procedures and portable simulators to use for distributed practice at home. Like other surgical specialties, ${ }^{3}$ procedures in cardiac surgery can be partitioned into components and lead to the development of partial-task trainers. After distributed practice, the residents in this study demonstrated improved performance in the task station or partial-task trainer, an improvement that was also evident in the beating-heart model.
For certain simulation tasks, a basic task station might not be able to distinguish between a junior trainee and an expert surgeon once the junior trainee has achieved a high level of competence ${ }^{6,11,16}$; that is, there might be a "ceiling effect" of certain simulators in the assessment of technical skills. ${ }^{6,11}$ Depending on the extent of previous training and experience, residents at the same training level might be at different proficiency levels ${ }^{15,16}$; as such, some residents might demonstrate no significant improvement after 1 week of distributed practice using a basic task station. Of the 2 residents who had no improvement using the task station, one was a third-year resident whose times to completion were the lowest and who had good performance rating scores. Given the resident's previous experience and his advanced technical level, this basic task station did not provide an adequate assessment of distributed practice. Conversely, one first-year resident demonstrated substantial improvement in the task station ( $49 \%$ decrease in time to completion); thus the task station is of greater utility in less experienced residents, which is consistent with previous findings regarding the use of low-fidelity bench models among novice surgeons. ${ }^{7}$ Interestingly, 2 third-year residents who had relatively low anastomosis times were still able to improve with practice; with intensive supervision, the skill level might further increase. Notably, the range of times for the 3 third-year residents in this study were comparatively low (216-270 seconds after practice), suggesting that the times to completion of the task station can be used as one component of proficiency assessment. $^{6}$

There might be a limit in the near term as to what a particular individual is able to improve. One first-year resident had a slight increase in time after 1 week of practice using the task station. On review of his performance by experienced surgeons, there were technical components that might be improved upon with additional coaching. Thus not only is there a ceiling effect in terms of the ability of a basic task simulator to improve a trainee's performance, there might be a "plateau effect" in terms of the ability of the resident to improve in the short term. Importantly, it is necessary to establish whether a trainee has achieved a plateau in technical ability 
for a given task by using simulation and whether additional coaching and practice could elevate the trainee to the next level.

Because clinical experience with beating-heart surgery remains less than that for arrested-heart surgery in our training program, the resident is less likely to be proficient with the former skill. Using the beating-heart model, the residents generally improved at 1 week after practice with the anastomosis task station. The novelty of and initial lack of experience with the beating-heart model might have contributed to the degree of improvement in performance after some use, particularly among third-year residents. However, 2 residents (1 at the second-year level and 1 at the first-year level) demonstrated no improvement with the beating-heart model. Although both residents demonstrated improvement in the task station after 1 week, 1 had moderate times to completion and the other had long times to completion using the beatingheart model, implying that both might benefit from additional coaching with the beating-heart model.

A limitation of this study is the relatively small number of residents evaluated, which is reflective of the small numbers of residents in cardiothoracic surgery training at 1 institution. A multi-institutional effort to evaluate novel training methods will be necessary to affect cardiac surgical curriculum development. Although such "dry-laboratory" simulation, such as the task station and the beating-heart model, can be valuable in training, disadvantages include the fact that it does not reproduce the tissue response seen with animal or human tissues. Also, because there are no contiguous vital or simulated structures, suspension of disbelief might be limited. The beating-heart model in the laboratory might provide a more realistic simulation and has been perceived by the residents to be a good method of training. Although intended to simulate coronary anastomosis on a beating heart, this model also can be used to simulate anastomosis on an arrested heart.

Finally, other modalities of cardiac surgery simulation have been used with success. Along with the anastomosis task station, we have developed aortic and mitral valve task stations and a "wet-laboratory" approach by using porcine hearts modeled after the wet-laboratory facility in the United Kingdom. ${ }^{23}$ We agree that less complex simulation exercises can be used to teach the technical aspects of cardiac surgery without the need to replicate the operating room environment. However, the addition of adverse conditions into the simulation scenarios, requiring the trainee to solve problems, might serve to strengthen the value of the training exercise, not only to perform under ideal conditions but also to rehearse responses to emergency situations. ${ }^{20}$ It is likely that a combination of synthetic tissue simulation, real tissue simulation (ie, "wet-laboratory" exercises), and environmental crisis management will provide the ideal concurrent simulation training in cardiothoracic surgery residency.
We acknowledge the generous advice and assistance of Lance Acree at Aviation Training Consulting, LLC, in Saverna Park, Maryland, and Christopher Munsch, ChM, FRCS, in Leeds, United Kingdom.

\section{References}

1. Rhodes RS. Defining general surgery and the core curriculum. Surg Clin North Am. 2004;84:1605-19.

2. Reznick RK, MacRae H. Teaching surgical skills—changes in the wind. $N$ Engl J Med. 2006;355:2664-9.

3. Reznick R, Regehr G, MacRae H, Martin J, McCulloch W. Testing technical skill via an innovative "bench station" examination. Am J Surg. 1996;172: 226-30.

4. Reznick RK. Teaching and testing technical skills. Am J Surg. 1993;165: 358-61.

5. Sutherland LM, Middleton PF, Anthony A, Hamdorf J, Cregan P, Scott D, et al. Surgical simulation: a systematic review. Ann Surg. 2006;243:291-300.

6. Hance J, Aggarwal R, Stanbridge R, Blauth C, Munz Y, Darzi A, et al. Objective assessment of technical skills in cardiac surgery. Eur J Cardiothorac Surg. 2005; 28:157-62.

7. Grober ED, Hamstra SJ, Wanzel KR, Reznick RK, Matsumoto ED, Sidhu RS, et al. The educational impact of bench model fidelity on the acquisition of technical skill. Ann Surg. 2004;240:374-81.

8. Moulton CA, Dubrowski A, MacRae H, Graham B, Grober E, Reznick R. Teaching surgical skills: what kind of practice makes perfect? Ann Surg. 2006;244 400-9.

9. Mackay S, Morgan P, Datta V, Chang A, Darzi A. Practice distribution in procedural skills training. Surg Endosc. 2002;16:957-61.

10. Datta V, Bann S, Beard J, Mandalia M, Darzi A. Comparison of bench test evaluations of surgical skill with live operating performance assessments. J Am Coll Surg. 2004;199:603-6.

11. Moorthy K, Munz Y, Adams S, Pandy V, Darzi A. A human factors analysis of technical and team skills among surgical trainees during procedural simulations in a simulated operating theatre. Ann Surg. 2005;242:631-9.

12. Wanzel KR, Matsumoto ED, Hamstra SJ, Anastakis DJ. Teaching technical skills: training on a simple, inexpensive, and portable model. Plast Reconstr Surg. 2002; 109:258-64.

13. Anastakis DJ, Regehr G, Reznick RK, Cusimano M, Murnaghan J, Brown M et al. Assessment of technical skills transfer from the bench training model to the human model. Am J Surg. 1999; 177:167-70.

14. Grantcharov TP, Kristiansen VB, Bendix J, Bardram L, Rosenberg J, FunchJensen R. Randomized clinical trial of virtual reality simulation for laparoscopic skills training. Br J Surg. 2004;91:146-50.

15. Seymour NE, Gallagher AG, Roman SA, O’Brien MK, Bansal VK, Andersen DK, et al. Virtual reality training improves operating room performance. Ann Surg. 2002;236:458-64

16. Brydges R, Kurahashi A, Brummer V, Satterthwaite L, Classen R. Developing criteria for proficiency-based training of surgical technical skills using simulation: Changes in performance as a function of training year. J Am Coll Surg. 2008; 206:205-11.

17. Stanbridge RD, O'Regan D, Cherian A, Ramanan R. Use of pulsatile beating heart model for training surgeons in beating heart surgery. Heart Surg Forum. 1999;2: 300-4.

18. Reuthebuch O, Lang A, Groscurth P, Lachat M, Turina M, Zund G. Advanced training model for beating heart coronary artery surgery: the Zurich heart-trainer. Eur J Cardiothorac Surg. 2002;22:244-8.

19. Bashar Izzat M, El-Zufari H, Yim APC. Training model for "beating-heart" coronary artery anastomoses. Ann Thorac Surg. 1998;66:580-1.

20. Ramphal PS, Coore DN, Craven MP, Forbes NF, Newman SM, Coye AA, et al. A high fidelity tissue-based cardiac surgical simulator. Eur J Cardiothorac Surg. 2005;27:910-6.

21. Gaba DM, Howard SK, Flanagan B, Smith BE, Fish KJ, Botney R. Assessment of clinical performance during simulated crises using both technical and behavioral ratings. Anesthesiology. 1998;89:8-18.

22. Donovan JJ, Radosevich DJ. A meta-analytic review of the distribution of practice effect: now you see it, now you don't. J Appl Psychol. 1999;84: 795-805.

23. Wet-Lab LTD. Available at: www.wetlab.co.uk. Accessed 15 May 2008. 